\title{
Comparison of Wheelbase Filtering Effect and Suspension Tuning Between Two- axle and Tri-axle Vehicle with Tandem Suspension
}

\author{
Fu Wenkui ${ }^{1}$, Ling Feng ${ }^{1,2}$, Peng Manlong ${ }^{1}$, Zhang Yunqing ${ }^{1}$ \\ 1. Huazhong University of Science \&Technology, Wuhan, China \\ 2. Wuhan Ordnance Noncommissioned Officer School, Wuhan, China \\ e-mail: mechust@163.com
}

\begin{abstract}
This paper presents a comparison of wheelbase filtering effect and suspension tuning between two-axle and triaxle vehicle. Interference angle, introduced to account for the conflict between bouncing motion and pitching motion, is used to study the responses of the tri-axle vehicle. Olley tuning, the recommended suspension design rule, is found to be not as suitable for suspension design of tri-axle vehicle with tandem suspension as for two-axle vehicle. At higher vehicle speeds, identical suspension design is effective to suppress pitching motion. Olley tuning is proved to be bad for pitching motion but good for the bounce response. At lower speeds, Olley tuning is an effective design to improve both pitch response and bounce response at natural frequency.
\end{abstract}

Keywords- Wheelbase filtering, Suspension tuning, Tri-axle, Interference angle

\section{INTRODUCTION}

Wheelbase filtering effect, a phenomenon caused by the time lag between the road inputs at the front and rear wheel, has a considerable effect on vehicle performance and makes vehicle dynamic analysis more complex [1]. When vehicle pitch dynamic is studied, wheelbase filtering effect is one of the main features to be discussed for its opposite effect on bounce and pitch performance. From the ride comfort perspective, human body is more sensitive to transverse vibration than vertical vibration. Therefore, pitching motion is considered annoying because it can cause transverse vibration [2].

Olley tuning, a suspension design guideline to inhibit pitching motion by making the rear suspension stiffer than the front, is widely accepted as a rule of practice [3]. Many literatures have dealt with Olley tuning to confirm the effectiveness of this design method to improve pitch ride. Best discovered Olley design would cause pitch suppression but with bounce reinforcement by applying random road excitation to a half-car model [4]. Crolla and King found that Olley design was not so effective to improve the pitch acceleration response as the displacement response [5]. Odhams concluded that Olley tuning provided a nearly optimal solution for minimizing horizontal acceleration at the driver's chest, while the vertical acceleraion was not optimal [6].

Sharp studied wheelbase filtering effect and Olley tuning for two-axle vehicle by calculating frequency response for the half-car over a wide range of speeds and design conditions [3]. The author commented that vehicle structural parameters are independent of wheelbase filtering effect. In order to describe the mechanism of Olley tuning, a nomenclature named 'interference angle' was introduced. Olley tuning was shown to be beneficial to pitch response at higher vehicle speeds whereas it was the opposite at lower speeds. Conclusion was made that Olley tuning was desirable for vehicle driving at higher speeds but not a worthwhile goal for driving at lower speeds [7].

Compared with a large number of literatures on wheelbase filtering effect and suspension tuning for two-axle vehicle, there are little publications on that for tri-axle vehicle. This paper established a tri-axle half-car model and obtained the frequency response to discuss the effect of Olley tuning on bounce ride and pitch ride performance.

\section{MODELling}

Both vehicle models of two-axle and tri-axle established in this paper are simplified as half-car models, which ignore roll motion of the sprung mass. In these half-car models, car body is modeled as a rigid body with two freedoms, which are pitch and bounce displacements. Each axle, which is also referred to as unsprung mass, has a bounce motion freedom. The tandem suspension of the tri-axle truck can be built equivalently as two connected suspensions, which are linked by a beam, which is called the load equalizer. The load equalizer rotates around the pivot in the car body to ensure that the rear two axles suffer from the equal ground forces. Because of the constraint of the revolute joint at the pivot, the load equalizer only has one rotation freedom. The bounce displacement of the load equalizer at the pivot location is equivalent to that of the rear end of the car body. Therefore, two-axle model has four freedoms in total, which are the front wheel bounce motion $\mathrm{z} 1$, rear wheel bounce motion $\mathrm{z} 3$, body bounce motion $\mathrm{zb}$ and pitch motion $\theta \mathrm{b}$. Tri-axle model has another two freedoms additionally, which are the middle wheel bounce motion and load equalizer rotation freedom. The half-car pitch plane models are shown diagrammatically in Figure 1.

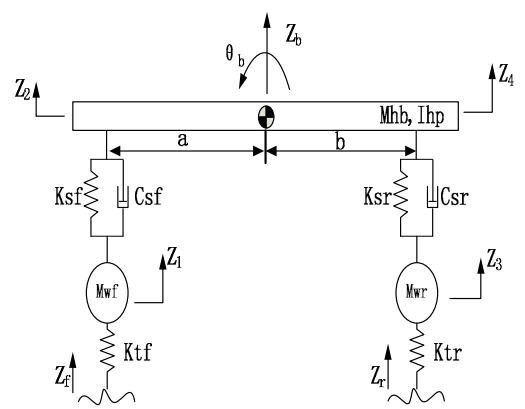

(a)Two-axle 


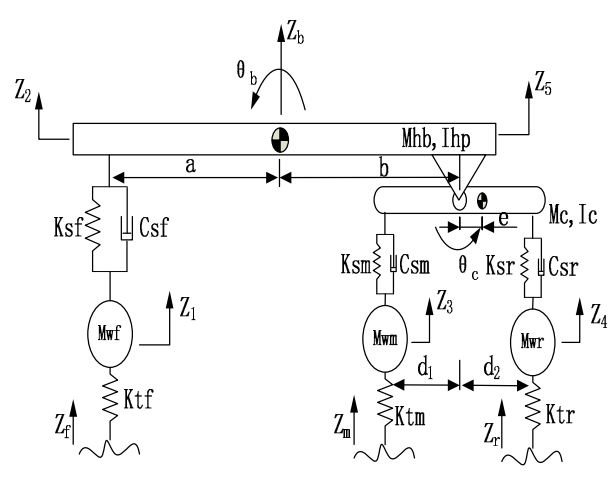

(b) Tri-axle

Figure 1. Half-car pitch plane models

\section{RESULTS AND THEIR INTERPRETATION}

In order to enhance the evidentness of the contrast between these two kinds of vehicles, the characteristic parameters of these two models are set to be identical and the front and rear suspensions are assumed to be symmetrical. System characteristic parameters are listed in Table I. Although the parameters differ greatly from those of the real heavy truck, it has some theoretical significance in qualitative analysis.

TABLE I. PARAMETER SETS OF TWO-AXLE AND TRI-AXLE

\begin{tabular}{|c|c|c|c|c|c|}
\hline $\begin{array}{l}\text { Parameter: } \\
\text { SI units }\end{array}$ & $\begin{array}{l}\text { Two- } \\
\text { axle }\end{array}$ & $\begin{array}{l}\text { Tri- } \\
\text { axle }\end{array}$ & $\begin{array}{l}\text { Parameter: } \\
\text { SI units }\end{array}$ & $\begin{array}{l}\text { Two- } \\
\text { axle }\end{array}$ & $\begin{array}{l}\text { Tri- } \\
\text { axle }\end{array}$ \\
\hline Mhb & 400 & 400 & Ktr & $\begin{array}{l}150 \\
000\end{array}$ & $\begin{array}{l}150 \\
000\end{array}$ \\
\hline Ihp & 625 & 625 & Mwf & 25 & 25 \\
\hline Ksf & $\begin{array}{c}16 \\
000\end{array}$ & $\begin{array}{c}16 \\
000\end{array}$ & Mwm & . & 25 \\
\hline Csf & 750 & 750 & Mwr & 25 & 25 \\
\hline Ksm & & $\begin{array}{c}16 \\
000\end{array}$ & Mc & $\longrightarrow$ & 10 \\
\hline Csm & - & 750 & Ic & - & 0.01 \\
\hline $\mathrm{Ksr}$ & $\begin{array}{c}16 \\
000\end{array}$ & $\begin{array}{c}16 \\
000\end{array}$ & $\mathrm{a}$ & 1.25 & 1.25 \\
\hline Csr & 750 & 750 & $\mathrm{~b}$ & 1.25 & 1.25 \\
\hline Ktf & $\begin{array}{l}150 \\
000\end{array}$ & $\begin{array}{l}150 \\
000\end{array}$ & d1 & 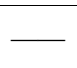 & 0.3 \\
\hline \multirow[t]{2}{*}{$\mathrm{Ktm}$} & & $\begin{array}{l}150 \\
000\end{array}$ & $\mathrm{~d} 2$ & 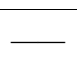 & 0.3 \\
\hline & & & $\mathrm{e}$ & $\longrightarrow$ & 0 \\
\hline
\end{tabular}

According to Sharp's statement, the interference angles of two-axle vehicle vary periodically on account of the wheelbase filtering effect. Noticablly, the sum of the interference angles of vertical acceleration and pitch angle/pitch acceleration equals $\pi$. It means when bouncing motion is maximum, pitching motion is minimum, and vice versa. Sharp took this conclusion as an explanation of Olley tuning. To show the effect of Olley tuning, an s factor is used as a multiplier for the rear suspension damper coefficient, s2 is the multiplier for the rear suspension spring stiffness and corresponding front coefficients are divided by the s and s2 factors.

Corresponding plots for tri-axle vehicle are given from Figure2 to Figure8. The wheel constant velocity are taken as the input for frequency response analysis. Interference angle is defined as the angle between the response excited by only front wheel input and response excited by middle and rear wheel input. Factor $s$ is also multiplied to the structural parameters of the middle suspension. Similarities between two-axle model and tri-axle model are listed as follows:

1.Sum of interference angle of both models equals $\pi$, which means the conflict between bouncing motion and pitching motion also exists in tri-axle model.

2.Changing of $s$ value has an obvious influence on the interference angles in the neighbourhood of natural frequency while the effect is slight at other frequencies.

The differences of these two models are concluded as follows:

1.Interference angles for tri-axle do not have a regular periodicity, as is shown in Figure2. Abnormal variations of intererence angles can be found at two different frequencies, One is located in low frequency, in the range of $1.5 \mathrm{~Hz}$ to 2 $\mathrm{Hz}$, and the other in high frequency, in the range of $16 \mathrm{~Hz}$ to $17 \mathrm{~Hz}$.

2.Olley tuning is not suitable for tri-axle model. As shown in Figure5, the pitch acceleration response of the original configuration is minimum compared with those of the other $\mathrm{s}$ values, which is different from the case in twoaxle vehicle. Conversely, increasing s will enlarge pitch acceleration response gain.
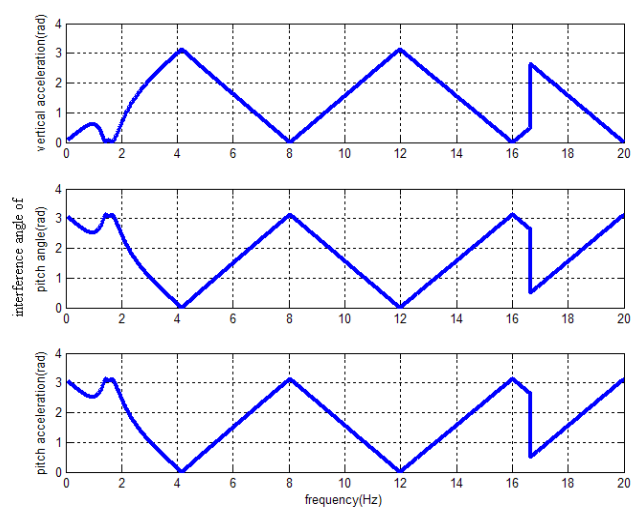

Figure 2. Interference angles of three responses for tri-axle model at 20 $\mathrm{m} / \mathrm{s}$

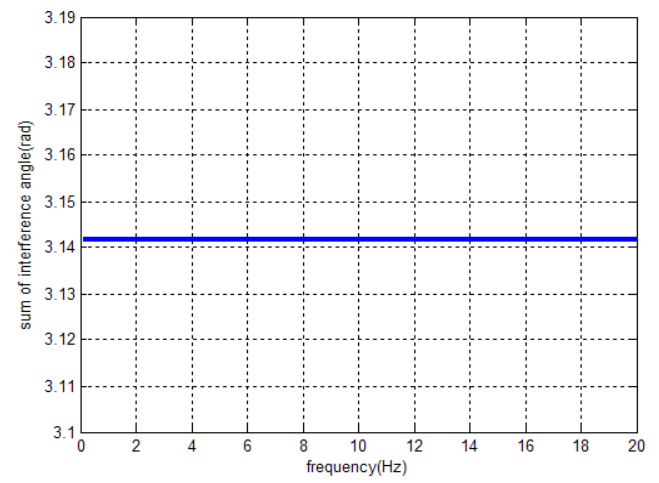

Figure 3. Sum of interference angles of vertical acceleration and pitch acceleration for tri-axle model at $20 \mathrm{~m} / \mathrm{s}$ 

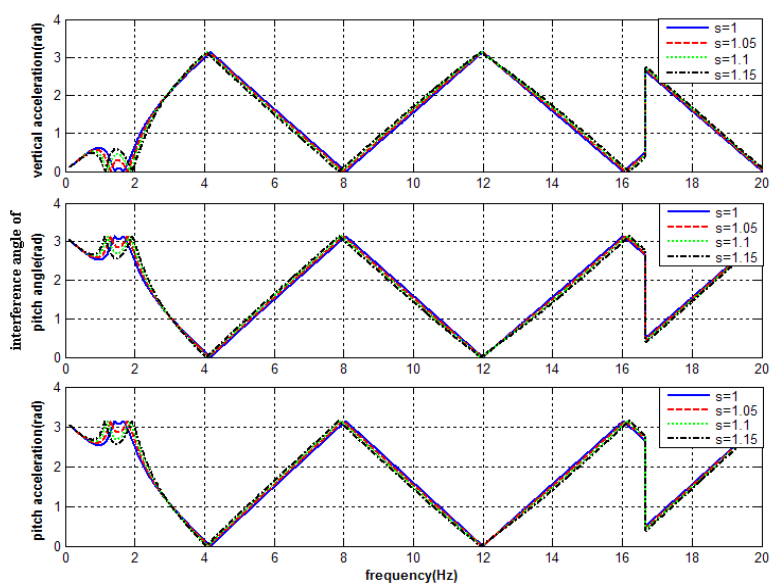

Figure 4. Interference angles of three responses for tri-axle model at 20 $\mathrm{m} / \mathrm{s}$ for different $\mathrm{s}$
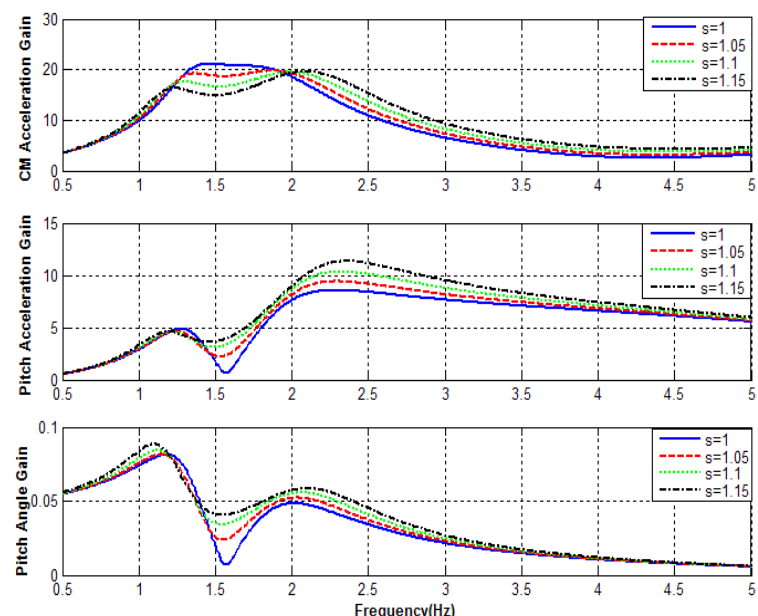

Figure 5. Frequency response results for tri-axle model at $20 \mathrm{~m} / \mathrm{s}$ for differents

Figure6 to Figure8 also present frequency responses from low speed to high speed. Effects of Olley tuning for tri-axle vehicle can be obtained in these figures. At low vehicle speeds, Olley tuning is an effective design to suppress both pitching motion and bouncing motion at natural frequency, demonstrated by Figure6 and Figure7. At higher speeds, original design is better for pitch response. Stiffening the middle and rear suspension will worsen pitching motion response and improve the bounce response.

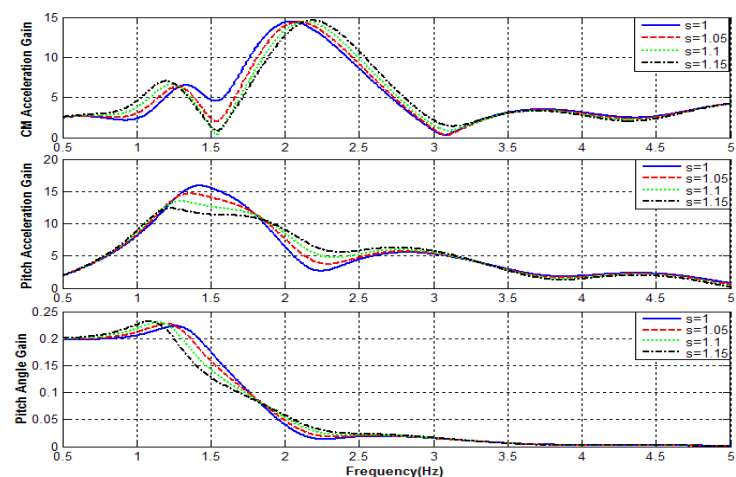

Figure 6. Frequency response results for tri-axle model at $5 \mathrm{~m} / \mathrm{s}$ for different s

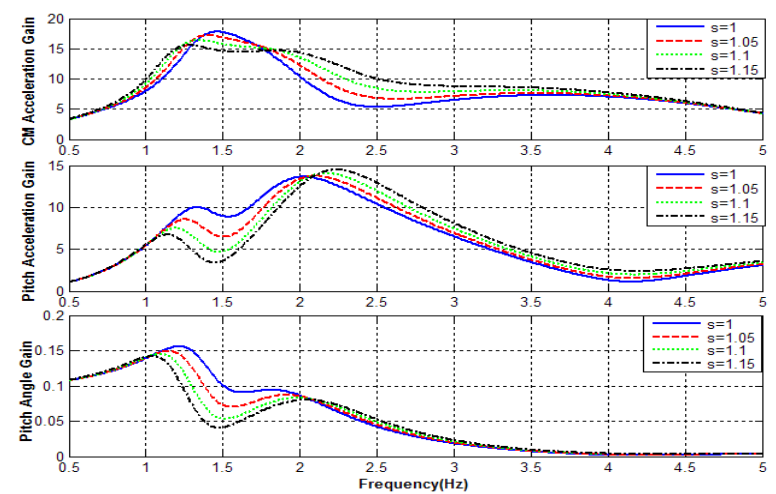

Figure 7. Frequency response results for tri-axle model at $10 \mathrm{~m} / \mathrm{s}$ for different $\mathrm{s}$

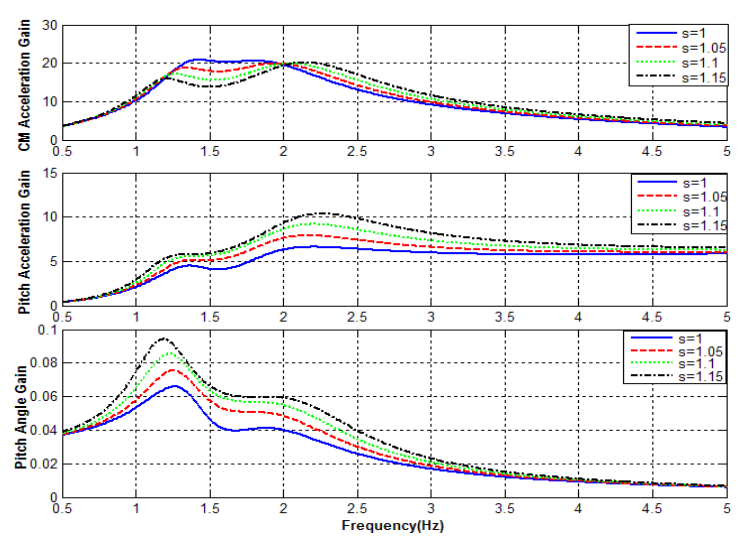

Figure 8. Frequency response results for tri-axle model at $30 \mathrm{~m} / \mathrm{s}$ for different $\mathrm{s}$

\section{CONCLUSION}

This paper compared the difference of wheelbase filtering effect between two-axle vehicle and tri-axle vehicle using half-car model. Interference angle was employed to show the conflict between pitching motion and bouncing motion. Frequency response of vertical acceleration, pitch acceleration and pitch angle were used as an evaluation to study Olley tuning. Olley tuning, which has been 
investigated previously and proven effective in improving the pitching motion response at higher speeds while not suitable in the lower speeds cases for two-axle vehicle, is extended into the tri-axle vehicle model. Results show that to improve the pitch response when the vehicle speed is high, it's better to choose a same type of suspension for the front and rear. In the lower speed cases, Olley tuning is an effective design to better both pitch ride and bounce ride performance at natural frequencies.

\section{REFERENCES}

[1] Dongpu Cao; Amir Khajepour; Xubin Song. Wheelbase Filtering and Characterization of Road Profiles for Vehicle Dynamics. IDET/CIE 2010,Canada
[2] Dongpu Cao; Xubin Song; Mehdi Ahmadian. Editors' Perspectives: Road Vehicle Suspension Design, Dynamics, and Control. Vehicle System Dynamics [J], 2011.49, PP: 3-28

[3] R.S Sharp. Wheelbase Filtering and Automobile Suspension Tuning for Minimizing Motions in Pitch. Automobile Engineering [J], 2002, PP:933-944

[4] Best, A. Vehicle Ride-stages in Comprehension. Phys. Technol [J], 1984.15, PP:205-210

[5] Crolla, D.A; King, R.P. Olley's 'flat ride' Revisited. Vehicle System Dynamics Supplement [J], 1999.33, PP:762-774

[6] Odhams, A.M.C; Cebon,D. An Analysis of ride coupling in automobile suspension. Automobile Engineering [J], 2006, PP:10411061

[7] R.S Sharp; Pilbeam,C. Achievability and value of passive suspension designs for minimum pitch response. Vehicle Ride and Handling, 1993.39, PP:243-259 